\title{
Post San Antonio Breast Cancer Symposium
}

\author{
Florian Fitzal
}

Received: 9 May 2017 / Accepted: 9 May 2017 / Published online: 30 May 2017

(C) Springer-Verlag Wien 2017

The 2016 SABCS (San Antonio Breast Cancer Symposium) provided delegates with a number of interesting and some praxis-changing data. This issue of memo highlights the most relevant data from the SABCS presented by leading breast cancer experts from Austria.

Locoregional issues are addressed by Michael Hubalek [1]. He presents data regarding sentinel node biopsy after neoadjuvant therapy from the GANEA trial in patients with clinical node-negative disease. Then Hubalek shows data with a protease-activatable radiometric fluorescent peptide dye conjugate (AVB$620)$ to identify malignant tissue intraoperatively and a poster from Denmark showing data of radioisotopic seed localisation technique for nonpalpable lesions. There were new interesting data regarding radiotherapy and breast reconstruction and a study regarding MRI for surgical planning.

Regarding systemic treatment in the adjuvant setting, Marija Balic [2] presents three prospective trials using extended endocrine therapy (DATA, IDEAL, NSABP-B42) with unexpected results, at least for some of us. The use of bisphosphonates in adjuvant therapy has been addressed in the TEAMIIb trial and Balic also presents data of patients with HER2-enriched breast cancer in the neoadjuvant (NSABP-B52; standard treatment with or without endocrine therapy) and in an adjuvant setting (only stage I; APT trial using paclitaxel weekly as backbone as well as adjuvant TDM1 versus trastuzumab using pertuzumab in both groups). Balic also shows a poster from the MINDACT trial comparing immunohistochemical with genomic subtype classification suggesting that the KI67 might

\section{Prof. Dr. F. Fitzal, MBA, F.E.B.S. (凶)}

Medical University for Surgery, Comprehensive Cancer

Center Vienna, Medizinische Universität Wien 21A,

Waehringer Guertel 18-20, 1090 Wien, Austria

florian.fitzal@meduniwien.ac.at be a good surrogate marker to differentiate genomic low from high risk.

Finally, Greil and Gampenrieder [3] highlighted data regarding palliative systemic treatment. The PrEGOC 0102 trial investigated whether the combination of fulvestrant plus everolimus is as safe and effective as exemestane plus everolimus for patients with hormone-receptor positive, HER2-negative disease. The pan-PI3K inhibitor buparlisib was used in combination with fulvestrant after failure of everolimus in the BELLE-3 trial. The BROCADE phase II trial added the PARP inhibitor veliparib to carboplatin and paclitaxel in patients with BRCA1/2 germline mutation. Finally, the TNT trial looked at biomarkers, in addition to BRCA1/2 germline mutation, for carboplatin benefit in patients with metastatic triple-negative breast cancer.

Wishing you an interesting and valuable reading.

Sincerely yours,

Univ. Prof. Dr. Florian Fitzal, F.E.B.S., MBA

Chief: Breast Surgery Unit

Department of Surgery

Medical University Vienna

Conflict of interest F. Fitzal declares that he has no competing interests.

\section{References}

1. Hubalek M, Czech T. Short review San Antonio 2016: Surgical therapies for breast cancer. Memo. 2017; doi 10.1007/ s12254-017-0331-7.

2. Suppan C, Balic M. Early stage breast cancer treatment and prognostic factors. Memo. 2017; doi 10.1007/s12254-0170328-2. 
3. Gampenrieder S, Rinnerthaler G, Greil R. SABCS 2016: systemic therapy for metastatic breast cancer. Memo. 2017; doi 10.1007/s12254-017-0326-4.

- For latest news from international oncology congresses see: http://www.springermedizin.at/ memo-inoncology 